\title{
Genome imprinting regulated by the mouse Polycomb group protein Eed
}

\author{
Jesse Mager ${ }^{1,2}$, Nathan D. Montgomery ${ }^{1,2}$, Fernando Pardo-Manuel de Villena ${ }^{1,3}$ \& Terry Magnuson ${ }^{1-3}$
}

Published online 10 March 2003; doi:10.1038/ng1125

\begin{abstract}
Epigenetic regulation is essential for temporal, tissue-specific and parent-of-origin-dependent gene expression. It has recently been found that the mouse Polycomb group (PcG) gene Eed (embryonic ectoderm development) acts to maintain repression of the imprinted $X$ chromosome. Here, we investigated whether Eed is also required for regulation of autosomal imprinted loci. Expression analyses showed that transcripts from the silent alleles of a subset of paternally repressed genes were present in $\mathrm{Eed}^{-/}$embryos. Parent-of-origin methylation was preserved in these embryos, but we observed changes in the methylation status of specific CpGs in differentially methylated regions (DMRs) at affected but not at unaffected loci. These data identify Eed as a member of a new class
\end{abstract} of trans-acting factors that regulate parent-of-origin expression at imprinted loci.

A subset of the mouse and human genomes is expressed from only one allele in a parent-oforigin-specific manner. This subset includes imprinted X-chromosome inactivation and autosomal imprinted loci. It has been proposed that this epigenetic regulation is accomplished through covalent modifications of both the DNA and the N-terminal tails of core histones in nucleosomes ${ }^{1,2}$. There are more than 60 identified autosomal imprinted genes, about half of which are paternally repressed and half maternally repressed. Most imprinted genes that have been examined contain at least one DMR located in the $5^{\prime}$ promoter region or in the body of the gene itself $^{3}$. Recently, several proteins (DNA methyltransferases, CpG methyl binding proteins, chromatin insulators) have been identified as trans-acting factors involved in the epigenetic regulation of these loci ${ }^{4}$. Many of these factors either possess or associate with proteins that possess DNA methyltransferase activity. Additionally, recent studies have shown correlations

Fig. 1 Expression patterns of imprinted genes in wild-type (wt) and $\mathrm{Eed}^{-1-}$ embryos. Representative results from individual embryos (each lane) are shown for each assay. m, maternal control; p, paternal control. a, RFLP analysis of $C d k n 1 c$ at E7.5 b. Typical results for direct sequencing of Ascl2 RT-PCR products. Arrowhead indicates base position of the expressed polymorphism (maternal $=$ A, paternal $=\mathrm{G}$ ). c, RFLP analysis of Grb10 at E7.5. $d$, RFLP analysis of Meg3 at E7.5. e, RFLP analysis of Kcnq1ot1 at E7.5. $f$, RFLP analysis of Kcnq1 at E7.5. $a$

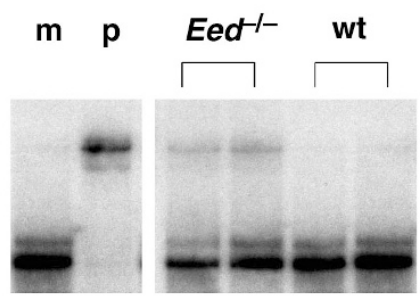

$c$

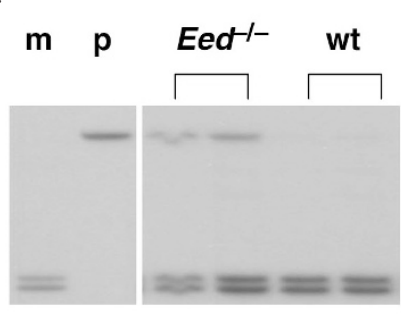

$e$

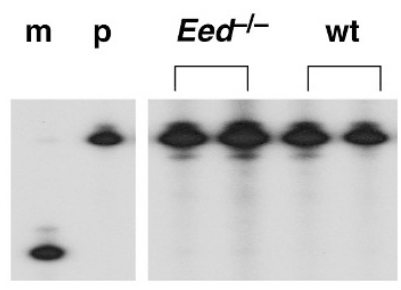

$b$

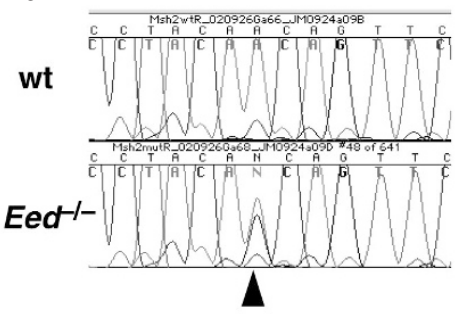

$d$

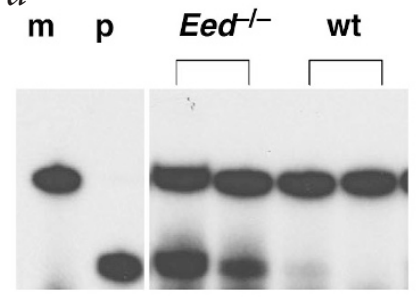

$f$

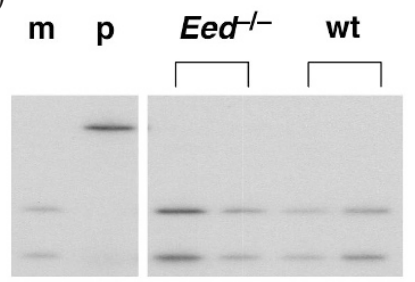

${ }^{1}$ Department of Genetics and Curriculum in Genetics and Molecular Biology, ${ }^{2}$ Carolina Center for Genome Sciences and ${ }^{3}$ Lineberger Comprehensive Cancer Center, University of North Carolina at Chapel Hill, 103 Mason Farm Road, Chapel Hill, North Carolina 27599-7264, USA. Correspondence should be addressed to T.M. (e-mail: terry_magnuson@med.unc.edu). 
results provide biochemical mechanisms by which the Eed/Ezh2 complex may establish heritable epigenetic states leading to long-term gene repression in vivo. We recently showed that loss of function of Eed in the mouse results in reactivation of the imprinted $\mathrm{X}$ chromosome in extraembryonic lineages ${ }^{12}$. Additionally, the Eed and Ezh2 proteins have been reported to co-localize with XIST on the imprinted X chromosome of mouse trophoblast stem cells ${ }^{13}$. Thus, we investigated whether Eed is also required for epigenetic regulation of autosomal imprinted loci.

We examined the expression patterns of 18 imprinted genes during peri-gastrulation stages (embryonic days (E) 5.5-8.5) using embryos derived either from an intersubspecific cross between Mus musculus domesticus (CD1) and Mus musculus molosinus (JF1) or from an intrasubspecific CD1 $\times$ C57BL/6 cross. For each gene examined, we identified a polymorphism in the mRNA to distinguish between maternal and paternal transcripts after RT-PCR. At all stages examined, the paternally repressed genes $C d k n 1 c$, Ascl2, Grb10 and Meg3 were expressed only from the maternal allele in wild-type mice (Fig. 1), showing that these four genes are imprinted during gastrulation. In

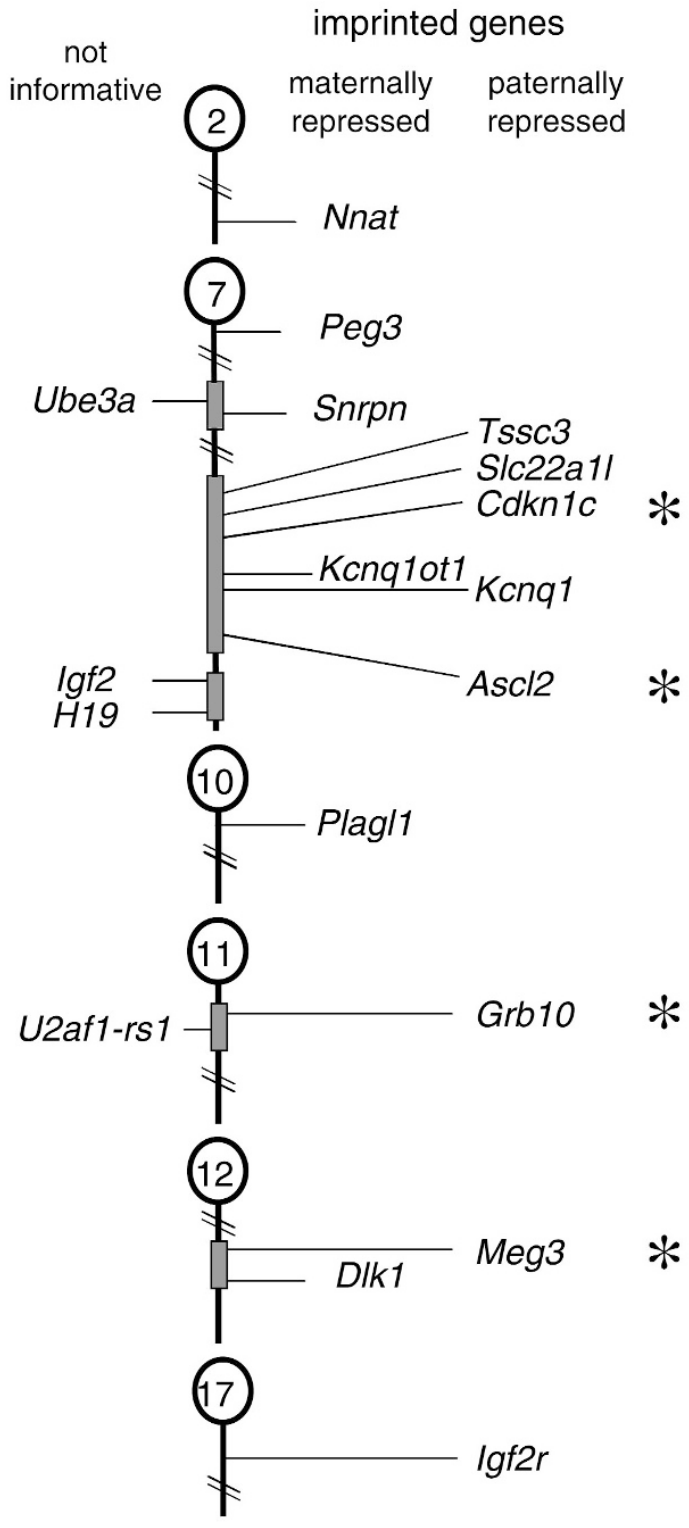

Eed ${ }^{17 R n 5-3354 \mathrm{SB}}$ null homozygous (herein referred to as $\mathrm{Eed}^{-/-}$) littermates, however, we found mRNA expression from both maternal and paternal alleles of these genes (Fig. $1 a-d$ ). Four other paternally repressed (Kcnq1, Slc22a1l, Tssc3 and Igf2r) and six maternally repressed genes (Kcnq1ot1, Snrpn, Peg3, Dlk1, Nnat and Plagl1) showed normal parent-of-origin expression patterns in $\mathrm{Eed}^{-1-}$ embryos (Figs. 1e,f and 2). These data indicate that loss of Eed function affects a subset of imprinted loci that are expressed early in development resulting in biallelic expression, herein referred to as loss of imprinting (LOI; ref. 14).

To determine whether LOI contributes to the early lethality of $\mathrm{Eed}^{-1-}$ embryos, we used a targeted null mutation of $C d k n 1 c^{15}$ to reduce $C d k n 1 c$ expression levels. Maternal inheritance of the $C d k n 1 c$ null allele resulted in $E e d^{-/-} C d k n 1 c^{+/-}$embryos that had developed substantially further than had $\mathrm{Eed}^{-1-} \mathrm{Cdkn1c^{+/+ }}$ embryos, although they were smaller and developmentally delayed when compared with wild-type littermates (data not shown). Morphological analysis at E8.5-9.0 showed that these embryos produced neural folds, a beating heart and somites, tissues that $\mathrm{Eed}^{-1-}$ embryos do not develop ${ }^{16}$. These data indicate that the LOI at Cdkn1c is partially responsible for the $\mathrm{Eed}^{-/}$ embryonic phenotype.

To address the molecular mechanisms leading to LOI at affected loci, we examined the methylation status of $\mathrm{CpG}$ islands at $C d k n 1 c, G r b 10, S n r p n$ and KvDMR1 by bisulfite sequencing of genomic DNA collected from E7.5 wild-type and $\mathrm{Eed}^{--}$ embryos. Analysis of a region $5^{\prime}$ to the transcriptional start site of Cdkn1c (CpG2; Fig. 3a) showed that the active maternal allele was hypomethylated and the inactive paternal allele was hypermethylated in wild-type embryos (Fig. $3 b, d$ ). These data extend previous observations from methyl-sensitive restriction digests that showed parent-of-origin methylation at three sites in the Cdknlc coding sequence ${ }^{17}$. In $\mathrm{Eed}^{-/-}$embryos, parent-of-origin methylation was present, but there were specific changes in the methylation pattern when compared with that of their wild-type counterparts (Fig. 3c). Eed ${ }^{-1-}$ maternal Cdkn1c alleles were unmethylated except for two CpGs at the $3^{\prime}$ end of the region analyzed. The $\mathrm{Eed}^{-l-}$ paternal $C d k n 1 c$ alleles also had a methylation pattern that was distinct from that of the wild-type paternal allele (Fig. 3c). Notably, the Eed ${ }^{-/-}$paternal Cdkn1c allele was active even though it was hypermethylated. This result was not anticipated because the silent paternal allele is hypermethylated in wild-type embryos. These data indicate that methylation at this DMR does not silence paternal Cdkn1c gene expression in $\mathrm{Eed}^{-1-}$ embryos. The specific pattern of CpG methylation, however, could be essential to the epigenetic state (inactive) of the paternal $C d k n 1 c$ allele. It is also notable that whereas there is parent-of-origin methylation in wild-type embryos at this DMR, it is not differentially methylated in germ cells, indicating post-fertilization regulation of methylation at this $\mathrm{CpG}$ island.

For Grb10 on mouse chromosome 11, we identified a potential DMR containing $146 \mathrm{CpG}$ dinucleotides in 1,200 bases located between exons 1 and 2. Bisulfite sequencing of 194 bases (Fig. 4a) containing $22 \mathrm{CpGs}$ confirmed parent-of-origin methylation at this $\mathrm{CpG}$ island. The active maternal allele was hypermethylated and the silent paternal allele was hypomethylated in wild-type embryos (Fig. $4 b$ ). Three consecutive CpGs

Fig. 2 Imprinted expression summary. Schematic representation indicating the chromosomal location of each of the 18 genes examined for imprinted expression in wild-type gastrulation-stage embryos. Ube3a, Igf2 and H19 showed biallelic expression, and U2af1-rs1 was not detected by RT-PCR; therefore, these genes were not informative. Maternally repressed genes and paternally repressed genes are listed in separate columns on the right side of each chromosome (number in centromere denotes chromosome). Genes that showed $\mathrm{LOI}$ in $\mathrm{Eed}^{-1-}$ embryos are marked with an asterisk. 
were always methylated (Fig. $4 c$ ) on the paternal allele in wildtype embryos, whereas all other CpGs were unmethylated. The $\mathrm{Eed}^{-1-}$ paternal Grb10 allele showed a variable pattern of hypomethylation, and the three CpGs that were invariantly methylated in wild-type embryos were not methylated in mutant embryos (Fig. 4c). We observed no differences between the wild-type and $\mathrm{Eed}^{-/-}$maternal Grb10 alleles, both of which were methylated at every $\mathrm{CpG}$ in the region analyzed. Similar analyses of the Snrpn DMR ${ }^{18}$, whose imprinted expression is not affected in $\mathrm{Eed}^{-/-}$embryos (Fig. 2), indicated no changes in parent-of-origin methylation patterns (Fig. $4 d$ ).

The imprinting control region (ICR) KvDMR1 on distal chromosome 7 has been shown to regulate several closely linked, paternally repressed genes including $C d k n 1 c$, Tssc3, Slca221l, Kcnq1 and Ascl2 (Fig. 5a), as paternal inheritance of a deletion of KvDMR1 results in LOI at these imprinted loci ${ }^{19}$. The observed LOI at these loci may be due to removal of the CpG island at the ICR or to disruption of the Kcnq1ot1 antisense transcript. To test the role of Eed at this ICR, we examined both parent-of-origin methylation at KvDMR1 (Fig. 5) and expression of the antisense Kcnq1ot1 transcript (Fig. 1f) in mutant embryos. Bisulfite sequencing in this ICR showed that parent-of-origin methylation was present in $\mathrm{Eed}^{-1}$ embryos. Maternal KvDMR1 alleles in both wild-type and $\mathrm{Eed}^{-1-}$ embryos were hypermethylated, but there were two CpGs that were never methylated in Eed $^{-1-}$ maternal KvDMR1 alleles but always methylated in wild-type maternal alleles (Fig. $5 b, c$ ). We recovered both methylated and unmethylated populations of paternal alleles from wild-type and $\mathrm{Eed}^{-/-}$littermates. Preliminary tissue-specific analysis suggests that the paternally methylated populations are predominantly extraembryonic in origin (data not shown). In the methylated paternal $K v D M R 1$ alleles, there was one

Fig. $3 C d k n 1 c$ CpG methylation. a, Schematic representation of a 10-kb genomic fragment of the Cdkn1c locus, indicating the location of exons (boxes 1-4) and CpG islands in the region (CpG1 and CpG2). CpG2 is expanded to indicate all 35 CpG dinucleotides (filled ovals) in the region analyzed after bisulfite treatment. The Smal restriction site used in $d$ is indicated. $\boldsymbol{b}, \boldsymbol{c}$ Parent-of-origin methylation patterns in wildtype (wt; b) and $\mathrm{Eed}^{-1-}$ (c) E7.5 embryos. m, maternal; $p$, paternal. Filled ovals indicate methylated CpGs; open ovals indicate unmethylated CpGs. Wild-type includes both $\mathrm{Eed}^{+/+}$and $\mathrm{Eed}^{+/-}$embryos. Symbols denote changes in the $\mathrm{Eed}^{-1-}$ methylation patterns. Arrows, gain of paternal-allele methylation; asterisks, loss of paternal-allele methylation; arrowheads, gain of maternal-allele methylation. $\boldsymbol{d}$, Genomic DNAs were digested with Smal before PCR and then analyzed by Tsp509I RFLP to assign parentof-origin to each of the alleles recovered through bisulfite sequencing, as there was no SNP in the region examined $(b, c)$. In both wild type and mutant embryos, only the paternal uncut PCR product (arrow) remained after Smal digestion, indicating that the paternal allele was methylated at this site (arrowhead in a) whereas the maternal allele was not. $m$, maternal; $p$ paternal. $\mathrm{F}$ and $\mathrm{R}$ indicate locations of forward and reverse PCR primers, respectively; Smal and Tsp509I restriction site locations are indicated in schematic ( $a$ and $d$ ).
CpG that was always methylated in wild-type embryos and never methylated in $\mathrm{Eed}^{-1-}$ embryos (Fig. 5c).

An insulator model for imprinted gene expression at this cluster has been proposed, on the basis of in vitro reporter assays that show CTCF, a zinc-finger protein, binds to unmethylated sites in the KvDMR1 $\mathrm{ICR}^{20}$. Alternatively, imprinted expression at this cluster could be regulated by the Kcnq1ot1 antisense transcript, similar to the model that has been proposed at the Igf2r/Air locus on chromosome 17 (ref. 21). Neither Kcnq1ot1 expression nor the methylation status of the ICR were altered in $\mathrm{Eed}^{-1-}$ embryos. These data indicate that Eed represents an additional mechanism of epigenetic regulation that is required to maintain parent-oforigin expression at specific loci in this cluster. In $\mathrm{Eed}^{--}$ embryos, Cdkn1c and Ascl2 had LOI but Slc22a1l, Tssc3 and Kcnq1 were properly imprinted, whereas all imprinted genes in the cluster were affected in the KvDMR1/Kcnq1ot1 deletion ${ }^{19}$. Gene-specific LOI was also observed in the cluster of imprinted genes on chromosome 12 (ref. 22), where Meg3 expression was subject to LOI but Dlk1 expression was properly imprinted in Eed $^{-1-}$ embryos. $a$
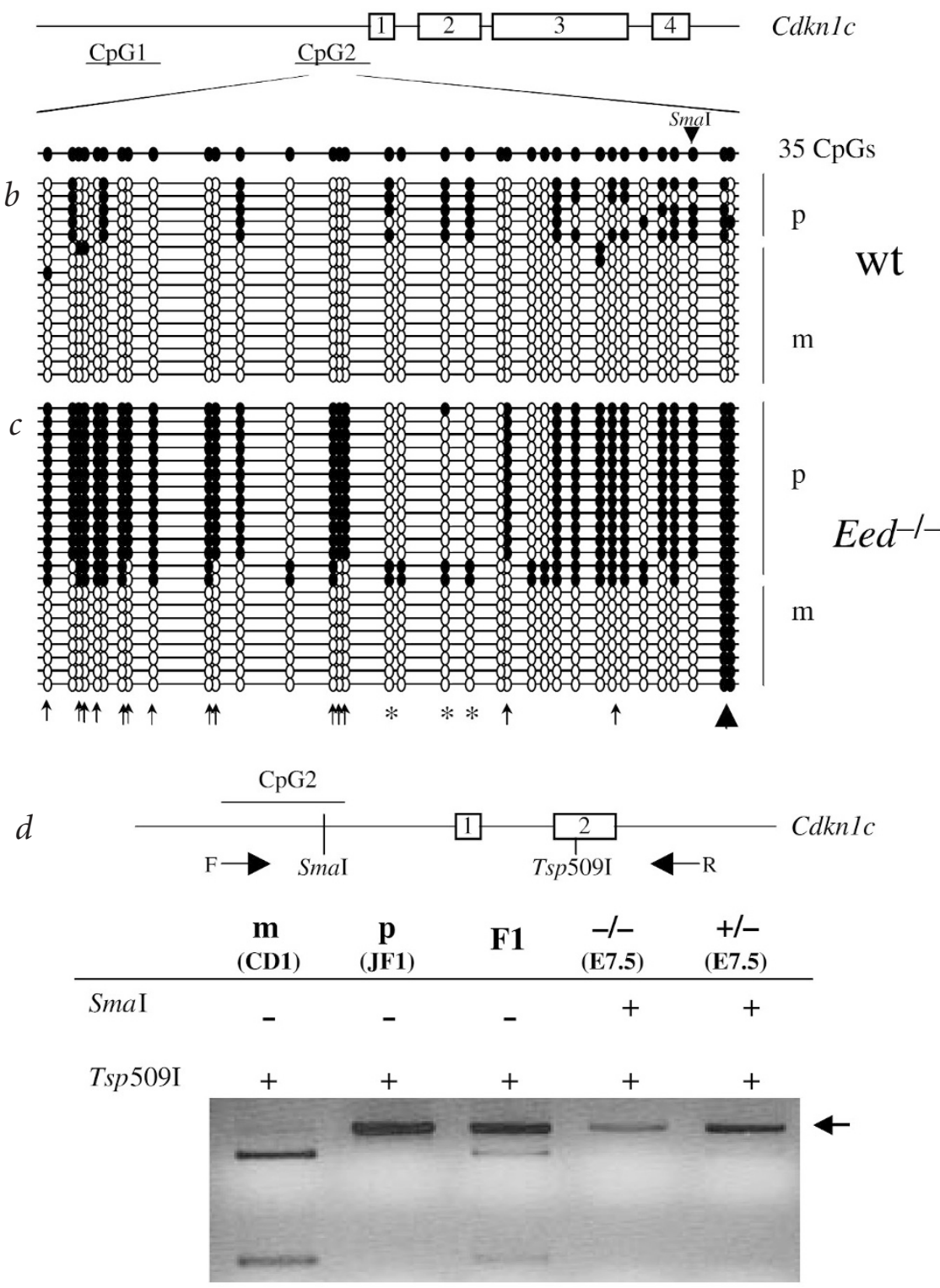
Our expression and methylation data identify Eed as a transacting factor that regulates imprinted gene silencing at a specific subset of autosomal loci during early development. Of the genes affected by mutations in Eed that have been identified thus far, no correlation with chromosomal location (Fig. 2) or methylation patterns was observed in wild-type embryos at ICRs or DMRs. For example, in normal littermates, the DMR of the active maternal $C d k n 1 c$ allele was unmethylated whereas at the DMR associated with Grb10, the active maternal allele was entirely methylated. The only consistent finding, including imprinted $\mathrm{X}$-chromosome inactivation ${ }^{12}$, is that in each case we observed biallelic expression of paternally repressed genes in mutant embryos (Fig. 2). Though these results are suggestive, more loci need to be examined to determine whether regulation by Eed is in fact restricted to paternally repressed genes.

DNA methyltransferase activity has not been associated with any $\mathrm{PcG}$ protein complexes. But it is possible that the alterations in DNA methylation patterns that we observed are intimately linked with altered covalent histone modifications.

$a$

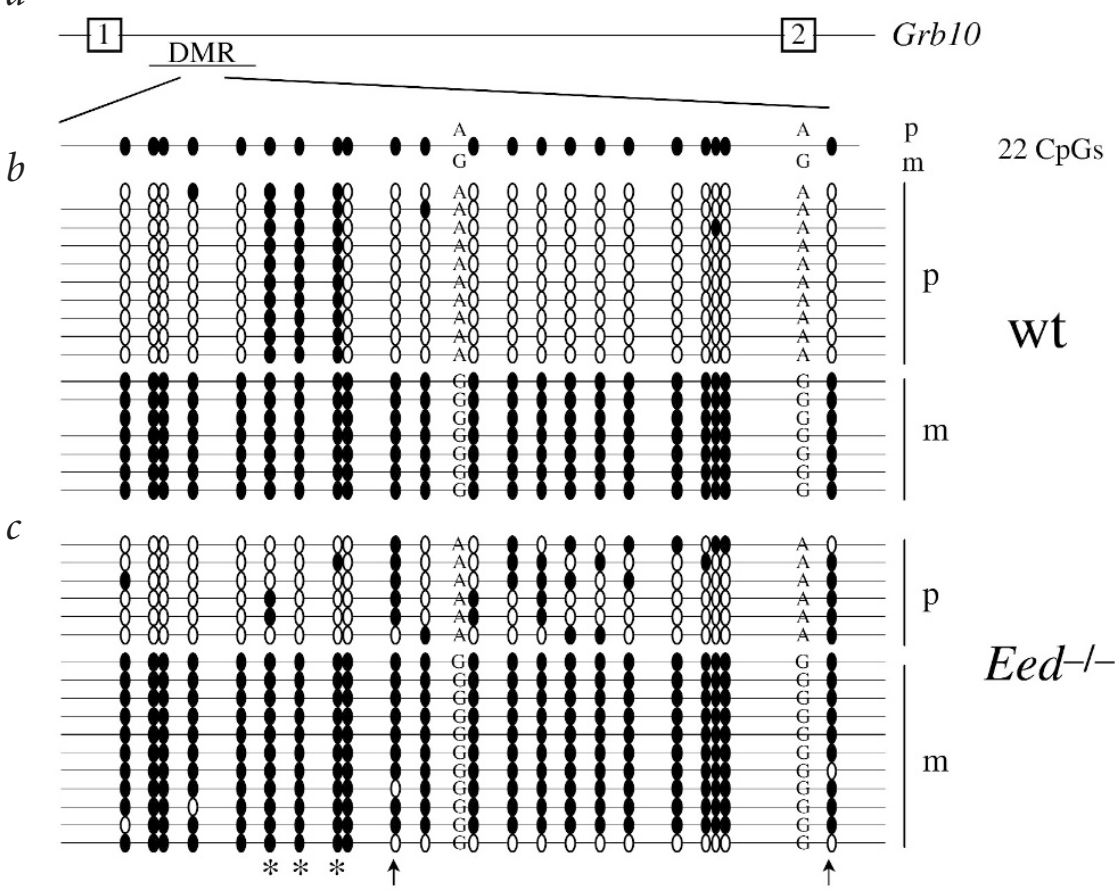

$d$

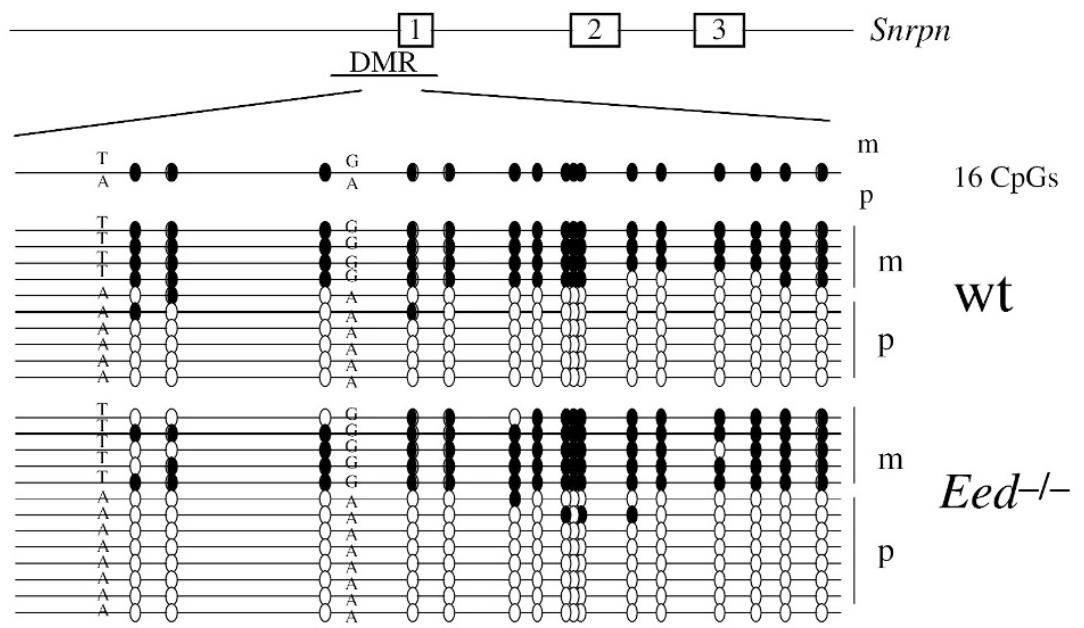

Recent studies in both Neurospora and Arabidopsis have shown that cytosine methylation is dependent on histone methylation ${ }^{23,24}$. Given the HMT activity of the Eed/Ezh2 complex and its potential for HDAC interactions, we propose that alterations to covalent histone modifications (methylation and possibly acetylation) are the primary defect resulting in LOI when the Eed/Ezh2 complex is disrupted and that the accompanying changes in DNA methylation patterns at Cdkn1c, Grb10 and KvDMR1 in Eed ${ }^{-1-}$ embryos are a secondary consequence.

The Eed/Ezh2 complex interacts through Eed with another PcG protein, Yy1, which has DNA-binding ability ${ }^{25}$. One possible explanation for the specificity of genes that show LOI in $\mathrm{Eed}^{--}$ embryos could be that Yy1 targets the Eed/Ezh2 complex to specific recognition sites of imprinted genes, resulting in local histone modifications. In the absence of functional Eed, Yyl may not be able to recruit the HMT activity of Ezh2, resulting in hypomethylation of histone $\mathrm{H} 3$ and a transcriptionally permissive environment leading to derepression of normally silent alleles. This model also explains the observed biallelic expression (as opposed to loss of expression of the active allele at affected loci) and is consistent with the role of Eed in a PcG group complex for maintaining long-term repression ${ }^{11}$.

At the affected loci examined, LOI cannot be the direct consequence of an inability of $\mathrm{Eed}^{-/-}$cells to distinguish between the parental chromosomes, as parent-of-origin methylation patterns were present in $\mathrm{Eed}^{-1-}$ embryos. This observation is in contrast with LOI caused by loss of parental imprints in uniparental disomies, deletion of ICRs or ablation of DNA methyltransferases. Our findings suggest that the presence of parent-of-origin methylation is not sufficient to direct imprinted gene expression at specific loci. It is possible, however, that particular patterns of CpG methylation may be essential for silencing of the paternal allele at affected loci and that the changes observed in Eed $^{-1-}$ embryos lead to LOI.

Fig. 4 Grb10 and Snrpn CpG methylation. a, Schematic representation of the genomic Grb10 locus (roughly $40 \mathrm{~kb}$ shown), indicating exons (boxes 1,2 ) and the location of the CpG island 4 kb $3^{\prime}$ of exon 1. The CpG island is expanded to show all $22 \mathrm{CpG}$ dinucleotides in the region analyzed after bisulfite treatment. Two SNPs in the region allowed for assignment of alleles recovered, and these are indicated by the base-pair changes $($ paternal $(J F 1)=A$, maternal $(C D 1)=G$ at each SNP). $\boldsymbol{b}, \boldsymbol{c}$, Parent-of-origin methylation patterns in wild-type (wt; b) and $\mathrm{Eed}^{-1-}(c)$ E7.5 embryos. Filled ovals indicate methylated CpGs; open ovals indicate unmethylated CpGs. Wildtype includes both $\mathrm{Eed}^{+/+}$and $\mathrm{Eed}^{+/-}$embryos. Symbols denote changes in the $\mathrm{Eed}^{-1-}$ methylation patterns. Arrows, gain of paternal-allele methylation; asterisks, loss of paternal-allele methylation; arrowheads, gain of maternalallele methylation. m, maternal; p, paternal. $\boldsymbol{d}$, Methylation analyses at the Snrpn promoter region. Filled ovals indicate methylated $\mathrm{CpGs}$; open ovals indicate unmethylated $\mathrm{CpGs}$. Wildtype includes both $\mathrm{Eed}^{+/+}$and $\mathrm{Eed}^{+/-}$embryos. m, maternal; $p$, paternal. 
Fig. 5 KvDMR1 CpG methylation. a, Schematic representation of genomic KVDMR1 locus indicating the six paternally repressed genes (boxes) that have been shown to be regulated by this ICR, located in intron 10 of Kcnq1 (ref. 28). KvDMR1 is expanded to indicate all 33 CpG dinucleotides in the region analyzed after bisulfite treatment. One SNP in the region allowed for assignment of alleles recovered, and these are indicated by the base-pair change (paternal (JF1) $=\mathrm{G}$, maternal $(C D 1)=\mathrm{T}$ ). One putative $C T C F$ binding site was identified containing 11 of 16 bases identical to conserved CTCF binding sites ${ }^{29}$. Two Eagl sites previously examined for parent-of-origin methylation are shown ${ }^{30} . \boldsymbol{b}, \boldsymbol{c}$, Parent-of-origin methylation at the ICR in wild-type (wt; b) and $\mathrm{Eed}^{-1-}$ (c) embryos. Note the presence of two populations of paternal alleles in both wild-type and $\mathrm{Eed}^{-1-}$ embryos. Filled ovals indicate methylated CpGs; open ovals indicate unmethylated $\mathrm{CpGs}$. Wild-type includes both $\mathrm{Eed}^{+/+}$and $\mathrm{Eed}^{+/-}$ embryos. Symbols denote changes in the $\mathrm{Eed}^{-1}$ methylation patterns. Asterisks, loss of paternalallele methylation; arrowheads, gain of maternal-allele methylation. $m$, maternal; $p$, paternal. Open circles, loss of maternal methylation.

Incorrect gene expression at imprinted loci has been reported in many human disorders, including Beckwith-Wiedemann syndrome, PraderWilli syndrome, Angelman syndrome and several types of cancer ${ }^{26}$. In a substantial fraction of individuals with these diseases, imprinting defects are observed in a subset of imprinted genes in the absence of any detectable muta-

tions in either the affected genes or known cis-acting regulatory elements ${ }^{14}$. Because Eed is required to maintain the epigenetic state leading to imprinted gene expression, we propose that mutations in EED or other trans-acting regulatory factors may account for the imprinting defects in these diseases. Here we showed that Eed is one such trans-acting factor that is necessary for parent-of-origin silencing of a subset of imprinted genes, providing the first link between a mammalian PcG gene and genome imprinting.

\section{Methods}

Expression analyses. We examined imprinted expression patterns by intron-spanning RT-PCR from freshly dissected gastrulation-stage embryos. We crossed $\mathrm{Eed}^{+/-} \mathrm{CD} 1$ dams with $\mathrm{Eed}^{+/-} \mathrm{JF} 1$ sires to produce embryos that were polymorphic at all loci examined except for Meg3 We used CD1 $\times$ C57BL/6 embryos to examine Meg3. For all expression analyses, we extracted mRNA from individual gastrulation-stage embryos using Invitrogen Tri-Reagent. We synthesized cDNA in $20-\mu$ reactions at $37^{\circ} \mathrm{C}$ for $1 \mathrm{~h}$ using Invitrogen SuperscriptII RT and then amplified it by 32 rounds of PCR with $1 \mathrm{mM} \mathrm{MgCl}_{2}$ at an annealing temperature of $60^{\circ} \mathrm{C}$. Polymorphism position (bp), SNP (CD1/JF1) and type of analysis used (enzyme) for each gene were as follows: Cdkn1c, bp 35,253 A/G, RFLP (Tsp509I); Dlk1, undetermined, bp 7,670-8,062, SSCP; Grb10, bp 897 G/A, RFLP (Cac8I); H19, bp 846 G/A, RFLP (BclI); Igf2, undetermined, bp 2,202-2,471, SSCP. Igf2r,13bp length polymorphism, bp 4,486-4,725; Kcnq1, bp 1,018 T/C, RFLP (AluI); Kcnq1ot1, bp 2,883 T/G, RFLP (HpyCH4IV); Ascl2, bp 1,315 T/C, direct sequencing; Meg3, bp 1,835 CD1 = G, B6 = A, RFLP (BsrDI); Nnat, undetermined bp 442-666, SSCP; Peg3, bp 5,329 A/C, RFLP (TaqI); Slc22a1l, bp 680 C/T, RFLP (NdeI); Snrpn, bp 391 (A/C), RFLP (NlaIII); Tssc3, bp 122 A/C, SSCP; Ube3a, bp 2,366 T/C, RFLP (Tsp509I); U2af1-rs1, undetermined, bp 4,966-5,381, SSCP; Plagl1, bp 941 A/G, RFLP (TaqI). Primer sequences are available on request. Al mice used were treated according to the recommendations of the Insti- tutional Animal Care and Use Committee of the University of North Carolina at Chapel Hill.

Bisulfite sequencing. After bisulfite treatment (as described in ref. 27), we carried out nested PCR reactions to amplify the following regions for methylation analysis: Cdkn1c, bp 34,033-34,328; Grb10, bp 65,194-65,398, SNPs at bp 65,297 and bp 65,230 (at both SNPs CD1 = C, JF1 = T); Snrpn, bp 67,729-68,105, SNP at bp 67,875 (CD1 = G, JF1 = A); KvDMR1, bp 2,542-2,934, SNP at bp 2,883 (CD1 = T, JF1 = G). Primer sequences are available on request. The conditions for first-round PCR were $3 \mathrm{~min}$ at $95^{\circ} \mathrm{C}$ and then 36 cycles of $45 \mathrm{~s}$ at $95^{\circ} \mathrm{C}, 56^{\circ} \mathrm{C}$ and $72{ }^{\circ} \mathrm{C}$. The conditions for second-round PCR were 3 min at $95^{\circ} \mathrm{C}$ and then 36 cycles of $45 \mathrm{~s}$ at $95^{\circ} \mathrm{C}, 60^{\circ} \mathrm{C}$ and $72^{\circ} \mathrm{C}$. All reactions included $10 \%$ dimethylsulfoxide and $1.5 \mathrm{mM} \mathrm{MgCl}_{2}$. We cloned PCR products from at least two independent reactions and sequenced individual clones from each sample. Bisulfite conversion efficiency ranged from $94.6 \%$ to $99.1 \%$ for independently treated samples. Between $59 \%$ and $81 \%$ of individual clones sequenced (range represents independent PCR reactions) showed unique patterns of bisulfite conversion (considering all cytosine residues).

Methylation-sensitive restriction digest at Cdkn1c. We first digested genomic DNAs from both mutant and heterozygous E7.5 embryos with the methyl-sensitive restriction enzyme SmaI and then carried out PCR analysis of a 1.4-kb segment (containing no additional SmaI sites; primer sequences available on request). We then digested the PCR products with Tsp509I for RFLP analysis and resolved them on a 0.5\% agarose gel ( Tsp509I polymorphism at position 35,253; CD1 = A, JF1 = G).

Accession numbers. Dlk1, AB047760; Grb10, NM010345; H19, NM023123; Igf2, 6754309; Igf2r, 20897376; Kcnq1, U70068; Kcnq1ot1, AF119385; Ascl2, BC019520; Meg3, Y13832; Nnat, AK003004; Peg3, AB003040; Slc22a1l, 6679179; Snrpn, 7705508; Tssc3, NM_009434; Ube3a, 27804320; U2af1-rs1, AF309654; Plagl1, AF147785; Cdkn1c, AP001293; Grb10, AL663087; Snrpn, AF332579; KvDMR1, AF119385. 


\section{Acknowledgments}

We thank D. Threadgill for providing the initial stock of JF1 mice and J. Trasler and T. Bestor for PCR primers and protocols. N.D.M. is supported by a predoctoral fellowship from the Howard Hughes Medical Institute. F.P.-M.V. is the recipient of a Junior Investigator award from the Andrew Mellon Foundation. This work was partially supported by the National Science Foundation to F.P.-M.V. and by the US National Institutes of Health to T.M.

\section{Competing interests statement}

The authors declare that they have no competing financial interests.

Received 4 October 2002; accepted 5 February 2003.

1. Strahl, B.D. \& Allis, C.D. The language of covalent histone modifications. Nature 403, 41-45 (2000)

2. Reik, W., Dean, W. \& Walter, J. Epigenetic reprogramming in mammalian development. Science 293, 1089-1093 (2001).

3. Strichman-Almashanu, L.Z. et al. A genome-wide screen for normally methylated human CpG islands that can identify novel imprinted genes. Genome Res. 12, 543-554 (2002).

4. Bird, A.P. \& Wolffe, A.P. Methylation-induced repression-belts, braces, and chromatin. Cell 99, 451-454 (1999)

5. Goll, M.G. \& Bestor, T.H. Histone modification and replacement in chromatin activation. Genes Dev. 16, 1739-1742 (2002).

6. Boggs, B.A. et al. Differentially methylated forms of histone $\mathrm{H} 3$ show unique association patterns with inactive human X chromosomes. Nat. Genet. 30, 73-76 (2002).

7. Pirrotta, V. Chromatin complexes regulating gene expression in Drosophila. Curr. Opin. Genet. Dev. 5, 466-472 (1995)

8. Cao, R. et al. Role of histone H3 lysine 27 methylation in Polycomb-group silencing. Science 298,1039-1043 (2002).

9. Czermin, B. et al. Drosophila enhancer of Zeste/ESC complexes have a histone $\mathrm{H}$ methyltransferase activity that marks chromosomal Polycomb sites. Cell 111 185-196 (2002).

10. Muller, J. et al. Histone methyltransferase activity of a Drosophila Polycomb group repressor complex. Cell 111, 197-208 (2002)

11. van der Vlag, J. \& Otte, A.P. Transcriptional repression mediated by the human Polycomb-group protein Eed involves histone deacetylation. Nat. Genet. 23 474-478 (1999).

12. Wang, J. et al. Imprinted $X$ inactivation maintained by a mouse Polycomb group gene. Nat. Genet. 28, 371-375 (2001).
13. Mak W et al. Mitotically stable association of Polycomb group proteins eed and enx 1 with the inactive $\mathrm{X}$ chromosome in trophoblast stem cells. Curr. Biol. 12, 1016-1020 (2002).

14. Nicholls, R.D. \& Knepper, J.L. Genome organization, function, and imprinting in Prader-Willi and Angelman syndromes. Annu. Rev. Genomics Hum. Genet. 2, 153-175 (2001).

15. Zhang, P. et al. Altered cell differentiation and proliferation in mice lacking p57KIP2 indicates a role in Beckwith-Wiedemann syndrome. Nature 387, 151-158 (1997).

16. Faust, C., Schumacher, A., Holdener, B. \& Magnuson, T. The eed mutation disrupts anterior mesoderm production in mice. Development 121, 273-285 (1995).

17. Hatada, I. \& Mukai, T. Genomic imprinting of p57KIP2, a cyclin-dependent kinase inhibitor, in mouse. Nat. Genet. 11, 204-206 (1995).

18. Bourc'his, D., Xu, G.L., Lin, C.S., Bollman, B. \& Bestor, T.H. Dnmt3L and the establishment of maternal genomic imprints. Science 294, 2536-2539 (2001).

19. Fitzpatrick, G.V., Soloway, P.D. \& Higgins, M.J. Regional loss of imprinting and growth deficiency in mice with a targeted deletion of KvDMR1. Nat. Genet. 32, 426-431 (2002).

20. Kanduri, C. et al. A differentially methylated imprinting control region within the Kcnq1 locus harbors a methylation-sensitive chromatin insulator. J. Biol. Chem. 277, $18106-18110$ (2002)

21. Sleutels, F., Zwart, R. \& Barlow, D.P. The non-coding Air RNA is required for silencing autosomal imprinted genes. Nature 415, 810-813 (2002).

22. Takada, S. et al. Epigenetic analysis of the Dlk1-Gtl2 imprinted domain on mouse chromosome 12: implications for imprinting control from comparison with Igf2-H19. Hum. Mol. Genet. 11, 77-86 (2002).

23. Jackson, J.P., Lindroth, A.M., Cao, X. \& Jacobsen, S.E. Control of CpNpG DNA methylation by the KRYPTONITE histone $\mathrm{H3}$ methyltransferase. Nature 416, 556-560 (2002).

24. Tamaru, H. \& Selker, E.U. A histone H3 methyltransferase controls DNA methylation in Neurospora crassa. Nature 414, 277-283 (2001).

25. Satijn, D.P., Hamer, K.M., den Blaauwen, J. \& Otte, A.P. The Polycomb group protein Eed interacts with $Y Y 1$, and both proteins induce neural tissue in Xenopus embryos. Mol. Cell. Biol. 21, 1360-1369 (2001).

26. Schofield, P.N. et al. Genomic imprinting and cancer: new paradigms in the genetics of neoplasia. Toxicol. Lett. 120, 151-160 (2001).

27. Tremblay, K.D., Duran, K.L. \& Bartolomei, M.S. A 5' 2-kilobase-pair region of the imprinted mouse $\mathrm{H} 19$ gene exhibits exclusive paternal methylation throughout development. Mol. Cell. Biol. 17, 4322-4329 (1997).

28. Horike, S. et al. Targeted disruption of the human LIT1 locus defines a putative imprinting control element playing an essential role in Beckwith-Wiedemann syndrome. Hum. Mol. Genet. 9, 2075-2083 (2000).

29. Farrell, C.M., West, A.G. \& Felsenfeld, G. Conserved CTCF insulator elements flank the mouse and human $\beta$-globin loci. Mol. Cell. Biol. 22, 3820-3831 (2002).

30. Smilinich, N.J. et al. A maternally methylated CpG island in KvLQT1 is associated with an antisense paternal transcript and loss of imprinting in Beckwith-Wiedemann syndrome. Proc. Natl. Acad. Sci. USA 96, 8064-8069 (1999). 